\title{
What Makes a Teen Get Tested? A Case of Urban Based Sample of Adolescents
}

Renata Arrington-Sanders ${ }^{1 *}$, Jonathan Ellen ${ }^{1}$, Roland $\mathbf{J}$ Thorpe $^{2}$ and Lori Leonard ${ }^{3}$

${ }^{1}$ Johns Hopkins School of Medicine, 200 North Wolfe Street, 2063, Baltimore, Maryland 21287, USA

${ }^{2}$ Assistant Professor, Department of Health Behavior and Society, Director, Program for Research on Men's Health, Hopkins Center for Health Disparities Solutions, Baltimore, MD, USA

${ }^{3} J o h n s$ Hopkins School of Public Health, 624 North Broadway, Room 298, Baltimore, Maryland 21205, USA

\begin{abstract}
Objective: Urban teens disproportionately impacted by HIV may not seek HIV testing. The objectives of this study are to determine factors that impact HIV testing among sexually active and non-sexually active teens seeking care in an urban academic setting; whether teens with high levels of self-efficacy are more likely to receive HIV testing; and whether an teen's ability to cope impacts positive attitudes toward testing

Methods: We conducted a cross-sectional survey of 228 HIV negative adolescent and young adult participants age 12-21 who received care in an academic urban primary care clinic in Baltimore, Maryland.

Results: Most youth reported being sexually active $(\mathrm{N}=146,64 \%)$ and reported having been tested at that day's visit $(\mathrm{N}=135,85 \%)$. Provider recommendation was significantly associated with higher odds of testing among sexually active teens (OR 3.5, 95\% $\mathrm{Cl} 1.07-11.7$ ) and those with no prior sexual history (OR 5.89, 95\% $\mathrm{Cl} 1.40-24.9$ ), while high HIV stigma was associated with lower odds of testing (OR $0.17,95 \% \mathrm{Cl} 0.04-0.77$ ) among youth with no prior sexual history. Sexually active teens with a positive attitude toward HIV testing were more likely to be older (late: $6.3(1.0-40)$ ), report intention to be tested in 6 months (OR 7.11, 95\% Cl 1.48-34.2), and have higher HIV coping self-efficacy (OR $1.12,95 \% \mathrm{Cl} 1.00-1.26)$.

Conclusions: Provider recommendation may be the most important independent factor for testing in teens, regardless of sexual history, while HIV-related stigma may be an important factor for teens with no prior sexual history and thus may be perceived to have little or no risk for HIV acquisition. In sexually active youth, older age, intention to be tested for HIV and the ability to cope with a positive diagnosis likely dictates adolescent attitudes toward engagement in HIV testing, although it may not directly correspond with HIV testing behavior.
\end{abstract}

Keywords: HIV testing; Adolescents; Teens; Coping self-efficacy

\section{Introduction}

Some urban teens disproportionately affected by HIV, are less likely to seek HIV testing and counseling, two essential components of HIV care [1,2]. In fact, less than $25-30 \%$ of urban African American youth report a history of HIV testing [1,2]. In order to effectively prevent and treat HIV in this population, we need better strategies to increase uptake of available HIV testing and counseling services.

Few explanations exist for why some teens living in urban communities engage in HIV testing while others do not. The small body of research examining adolescent HIV testing has found that not being offered a test is one major reason teens state for not being tested for HIV [3]. Studies have also demonstrated that stigma inhibits and social support facilitates test acceptance in low-income urban teens $[4,5]$.

HIV stigma has been defined as "the prejudice, discounting, discrediting, and discrimination related to HIV/AIDS and has been directed at people perceived to have HIV/AIDS [6]. In teens, HIV and STD stigma has been associated with decreased testing behavior because of the perception that others associate adverse judgment to testing and diagnosis [5].

Low perceived risk, "fear of being positive" and being "unable to deal with" a positive diagnosis are other important reasons for not being tested [3]. One key motivator for whether an urban adolescent is tested for HIV may be general self-efficacy, or the belief that one can succeed in specific situations [7]. Self-efficacy reflects a teens' ability to master specific activities, situations or aspects of psychological and social functioning [8]. Teens who live in low-income urban communities may perceive decreased personal control, and they may only regain a sense of control after they have mastered skills and learned to rely on themselves [7]. One recent study found an association with history of HIV testing and greater general self-efficacy among inner city black teens [7]. Coping with an HIV positive diagnosis may, therefore, depend on having attitudes that one has the mastery of skills necessary to receive a positive diagnosis.

Coping self-efficacy, defined as confidence in one's ability to cope with an HIV infection, may be especially relevant to HIV testing attitudes and behavior in urban teens $[5,10]$. In other populations, such as the homeless, pregnant women and incarcerated men in Jamaica, coping self-efficacy was found to be an important factor in HIV test acceptance [11-13]. Furthermore, research has demonstrated that youth living in low-income communities who are exposed to acute and chronic stress are more likely to use strategies such as coping and social support to support healthy development and to buffer the negative effects of environmental stress [14].

*Corresponding author: Renata Arrington-Sanders, Johns Hopkins School of Medicine, 200 North Wolfe Street, 2063, Baltimore, Maryland 21287, USA, Tel: 410-502-8166; Fax: 410-502-5440; E-mail: rarring3@jhmi.edu

Received June 17, 2014; Accepted June 29, 2014; Published August 12, 2014

Citation: Arrington-Sanders R, Ellen J, Thorpe RJ, Leonard L (2014) What Makes a Teen Get Tested? A Case of Urban Based Sample of Adolescents. J AIDS Clin Res 5: 336. doi:10.4172/2155-6113.1000336

Copyright: (c) 2014 Arrington-Sanders R, et al. This is an open-access article distributed under the terms of the Creative Commons Attribution License, which permits unrestricted use, distribution, and reproduction in any medium, provided the original author and source are credited. 
Despite links between self-efficacy, coping self-efficacy, and HIV testing, there is little work examining the use of coping in urban AfricanAmerican teens who are disproportionately exposed to individual and community level stressors like poverty, community violence, drugs and poor education $[15,16]$. We do not know whether presence of coping self-efficacy (ability to cope with a positive HIV diagnosis) influences attitudes and behavior toward routine HIV testing.

The purpose of this study was to determine 1) factors that impact HIV testing among sexually active and non-sexually active teens seeking care in an urban setting disproportionately affected by HIV; 2) whether coping impacts positive attitudes toward testing; and 3) whether teens use HIV coping self-efficacy as a strategy to receive HIV testing as part of routine clinical care.

\section{Methods}

A cross-sectional sample of 249 teens and young adult participants age 12-21 (mean=16; $\mathrm{SD}=2.3$ ) receiving routine primary care (physical exams, etc.) in an urban primary care clinic completed a confidential survey at the end of the visit about routine HIV testing between January-May 2008. This age range was chosen because it represents a standard age range of adolescence in the U.S. We calculated a sample size based on the ability to detect a $15 \%$ difference in stigma (as a marker of HIV testing self-efficacy) between those who tested and those who declined testing. This strategy has been used in prior research [17]. Active consent was obtained from participants $\geq 18$ years or parents/ guardians of participants $<18$ years (with adolescent assent). Active consent requires participants or parents to sign a consent form prior to participating in the study. Participants received a remuneration of $\$ 10$ for the time to complete the survey. The institutional review board at the Johns Hopkins School of Public Health approved all study procedures.

Participants were recruited from a clinic in Baltimore, Maryland, a city that has an estimated community HIV incidence of 35.4 per 100,000 persons that occurs predominantly among African American men who have sex with men and heterosexual females [18]. Participants completed a 20 item self-administered survey developed from prior studies assessing HIV risk. Eight percent $(n=21)$ of subjects self-reported being HIV positive and were excluded from this study. The final analytic sample includes HIV negative subjects $(n=228)$ who submitted complete data reporting prior HIV testing history, receipt of HIV test at the visit, HIV risk, and intention to be tested in 6 months.

Participants reported level of agreement on an HIV coping selfefficacy (alpha $=0.86)$ and HIV testing stigma $(\alpha=0.83)$ scale developed by Andrinopoulos et al. [13] and adapted for use in teens. Each scale was a 7 item, 4-point Likert scale (from "strongly agree" to "strongly disagree").
Positive attitude toward routine HIV testing was assessed by answering "yes" to both of the following questions: "HIV should be a standard part of your care, just like taking your blood pressure or checking your weight"; "Everyone should be tested for HIV when they come to the clinic".

Reliability analysis was conducted for each aggregate measure using the statistical software SPSS Version 17.0(SPSS, Inc., Chicago, Illinois). Chi square tests and ANOVA were used to examine the proportional and mean differences between sexual activity status and select characteristics of the sample. The association between individual characteristics and HIV testing, self-efficacy and HIV testing, and teens' ability to cope and attitudes towards HIV testing were examined. Variables associated with routine HIV testing were explored using simple and multiple logistic regression analyses. Variables significant at the p-value $<0.10$ in simple logistic regression and that could theoretically impact testing behavior and attitudes toward testing behavior were included in multiple logistic regression. We used a liberal p-value of $<0.10$ to avoid exclusion of potentially important variables. STATA Intercooled Version 11.0 was used to conduct the logistic regression models. (Stata, Corp., College Station, TX).

\section{Results}

Characteristics of participants in the study sample are displayed in Table 1. Most youth reported being sexually active ( $\mathrm{N}=146,64 \%)$. Sexually active teens were more likely to be older, to report higher number of lifetime partners, to report prior STD diagnosis/HIV testing history and more likely to be tested for HIV in the next 6 months than those teens who are not sexually active. There were no differences observed between sexually active teens and those who are not sexually active with respect to being female or African American.

\section{Attitudes toward routine HIV testing}

Factors associated with positive attitude toward routine HIV testing are shown in Table 2 . Most sexually active youth who completed the survey reported a positive attitude toward routine HIV testing $(\mathrm{N}=135$, 92\%). Among this group, teens with a positive attitude toward HIV testing were more likely to be older age, report an intention to be tested in 6 months, and had been previously tested for HIV. There was a trend that a positive attitude towards HIV testing was associated with higher HIV coping self-efficacy and a prior STD diagnosis. After controlling for significant covariates, positive attitudes toward HIV testing were significantly associated with older age, intention to be tested in 6 months, and greater coping self-efficacy.

While most ( $\mathrm{N}=69,83 \%)$ teens who reported no prior sexual history described a positive attitude toward routine HIV testing, few factors

\begin{tabular}{|c|c|c|}
\hline Characteristic & $\begin{array}{l}\text { Sexually Active (N=146) } \\
\% \text { (N) or Mean (SD, Range) }\end{array}$ & $\begin{array}{l}\text { Not Sexually Active }(\mathrm{N}=83) \\
\%(\mathrm{~N}) \text { or Mean (SD, Range) }\end{array}$ \\
\hline Female Gender & $74 \%(108)$ & $65 \%(54)$ \\
\hline African American Race & $93 \%(136)$ & $90 \%(75)$ \\
\hline \multicolumn{3}{|l|}{ Age } \\
\hline $11-14$ & $12 \%(17)$ & $51 \%(42)^{*}$ \\
\hline $15-16$ & $30 \%(44)$ & $33 \%(27)^{*}$ \\
\hline $17+$ & $56 \%(86)$ & $17 \%(14)^{*}$ \\
\hline Lifetime Sex Partners & 5.0 (S.D. 5.51, Range: 1-28) & 0.17 (S.D. 1.03 , Range: 0-9)* \\
\hline History of prior STD diagnosis & $32 \%(47)$ & $2 \%(2)^{*}$ \\
\hline Intend to be tested in 6 months & $79 \%(111)$ & $32 \%(27)^{*}$ \\
\hline Tested prior for HIV & $73 \%(106)$ & $17 \%(14)^{*}$ \\
\hline
\end{tabular}

Table 1: Select Characteristics of the Sample. 
Citation: Arrington-Sanders R, Ellen J, Thorpe RJ, Leonard L (2014) What Makes a Teen Get Tested? A Case of Urban Based Sample of Adolescents. J AIDS Clin Res 5: 336. doi:10.4172/2155-6113.1000336

Page 3 of 5

\begin{tabular}{|c|c|c|c|}
\hline Characteristic & UAOR & $95 \% \mathrm{Cl}$ & MLR OR (CI)* \\
\hline Female Gender & 2.12 & $0.63-7.13$ & \\
\hline African American Race & 2.43 & $0.94-6.28$ & \\
\hline Lifetime Sex Partners & 1.14 & $0.92-1.41$ & \\
\hline \multicolumn{4}{|l|}{ Age } \\
\hline $11-14$ & --- & & \\
\hline $15-16$ & 5.69 & $1.19-27.4$ & \\
\hline $17+$ & 8.54 & $2.0-36.3$ & $6.29(1.00-39.5)$ \\
\hline History of prior STD diagnosis & 5.87 & $0.74-46.9$ & \\
\hline Tested prior for HIV & 4.28 & $1.27-14.4$ & \\
\hline Provider recommended testing & 1.37 & $0.41-4.54$ & \\
\hline Intend to be tested in 6 months & 9.72 & $2.69-35.2$ & $7.11(1.48-34.2)$ \\
\hline High HIV testing stigma ${ }^{\approx}$ & 0.43 & $0.13-1.42$ & \\
\hline HIV coping self-efficacy $\approx$ & 1.08 & $1.0-1.19$ & $1.12(1.00-1.26)$ \\
\hline \multicolumn{4}{|c|}{ 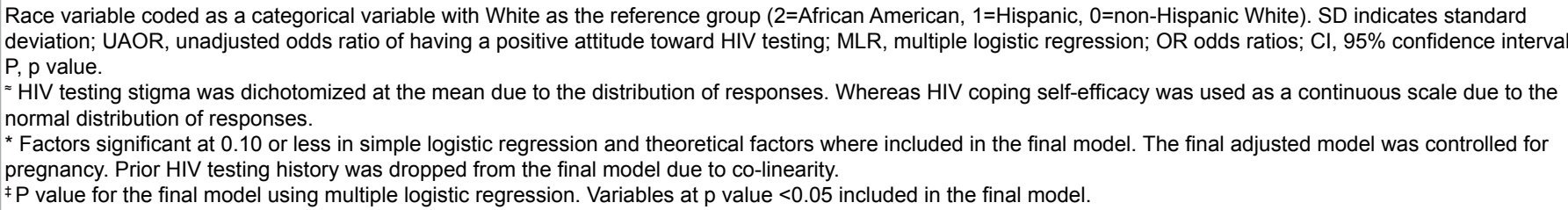 } \\
\hline
\end{tabular}

Table 2: Factors Associated with Positive Attitude toward Routine HIV Testing in Sexually Active Adolescents ( $N=135,92 \%)$.

\begin{tabular}{|c|c|c|c|}
\hline Characteristic & UAOR & $95 \% \mathrm{Cl}$ & MLR OR $(95 \% \mathrm{Cl})^{*}$ \\
\hline Female Gender & 3.11 & $1.24-7.80$ & $3.73(1.07-12.9)$ \\
\hline African American Race & 2.0 & $0.45-8.90$ & \\
\hline Lifetime Sex Partners & 1.45 & $1.09-1.92$ & \\
\hline \multicolumn{4}{|l|}{ Age } \\
\hline $11-14$ & (ref) & & \\
\hline $15-16$ & 4.9 & $1.33-18.30$ & \\
\hline $17+$ & 5.9 & $1.80-19.40$ & \\
\hline History of prior STD diagnosis & 13.1 & $1.74-100$ & \\
\hline Tested prior for HIV & --- & ---- & \\
\hline Provider recommended testing & 3.6 & $1.42-9.0$ & $3.23(1.02-10.3)$ \\
\hline Intend to be tested in 6 months & 5.6 & $2.1-14.7$ & \\
\hline High HIV testing stigma ${ }^{\approx}$ & 0.35 & $0.14-0.88$ & \\
\hline HIV coping self-efficacy ${ }^{\approx}$ & 1.0 & $0.92-1.07$ & \\
\hline \multicolumn{4}{|c|}{$\begin{array}{l}\text { Race variable coded as a categorical variable with White as the reference group ( } 2=\text { African American, } 1=\text { Hispanic, } 0=\text { non-Hispanic White). SD indicates standard } \\
\text { deviation; UAOR, unadjusted odds ratio of having a positive attitude toward HIV testing; MLR, multiple logistic regression; OR odds ratios; CI, } 95 \% \text { confidence interva } \\
\text { P, p value. } \\
\approx \text { HIV testing stigma was dichotomized at the mean due to the distribution of responses. Whereas HIV coping self-efficacy was used as a continuous scale due to the } \\
\text { normal distribution of responses. } \\
{ }^{*} \text { Factors significant at } 0.10 \text { or less in simple logistic regression and theoretical factors where included in the final model. The final adjusted model was controlled for } \\
\text { pregnancy. Prior HIV testing history was dropped from the final model due to co-linearity. } \\
\text { ₹P value for the final model using multiple logistic regressions. Variables at } p \text { value }<0.05 \text { included in the final model. } \\
\text { E Range of self-reported number of partners among youth who reported being not sexually active }(0-9) \text {. }\end{array}$} \\
\hline
\end{tabular}

Table 3: Factors Associated with HIV Testing in Sexually Active Adolescents ( $N=123,84 \%)$.

were associated with positive attitude toward routine testing. Intention to be tested in the next 6 months (OR 7.9, 95\% CI 1.0-64.4) was the only factor associated with positive attitude toward HIV testing.

\section{HIV testing behavior}

The association between selected characteristics and HIV testing is shown in Table 3. Among sexually active teens, most $(\mathrm{N}=123,84 \%)$ reported being tested as part of their health maintenance visit. Receipt of an HIV test at the visit was associated with female gender, higher reported lifetime sexual partners, older age, prior STD diagnosis, provider recommendation, and intends to be tested in the next 6 months. A lower odd of testing was associated with higher HIV stigma. Reporting same gender partner had no affect on the odds of receiving a test at the visit.

After adjusting for significant covariates and a potential confounder (pregnancy), in the final adjusted model, female gender and provider recommendation were the only variables that significantly associated with higher odds of testing in the sexually active teens. There was a trend that higher number of lifetime sex partners was associated with higher odds of receipt of an HIV test (OR 1.32, 95\% CI 0.99-1.74), but this did not remain significant in the final model.

Among youth with no prior sexual history ( $\mathrm{N}=83,36 \%), 22 \%(\mathrm{~N}=18)$ were tested for HIV at the visit. Receipt of an HIV test was associated with provider recommendation of the test and intention to be tested, while a lower odd of testing was associated with high HIV stigma (Table 4). After adjusting for significant covariates, in the final adjusted model (Table 4), provider recommendation was independently associated with higher odds of testing and high HIV stigma was associated with lower odds of testing. 


\begin{tabular}{|c|c|c|c|}
\hline Characteristic & UAOR & $95 \% \mathrm{Cl}$ & MLR OR $(95 \% \mathrm{Cl})^{*}$ \\
\hline Female Gender & 2.2 & $0.65-7.40$ & \\
\hline African American Race & 0.35 & $0.11-1.13$ & \\
\hline Lifetime Sex Partners ${ }^{£}$ & 1.06 & $0.67-1.67$ & \\
\hline \multicolumn{4}{|l|}{ Age } \\
\hline $11-14$ & (ref) & & \\
\hline $15-16$ & 1.1 & $0.32-4.03$ & \\
\hline $17+$ & 3.75 & $0.99-14.2$ & \\
\hline History of prior STD diagnosis & 3.76 & $0.22-63.3$ & \\
\hline Tested prior for HIV & --- & --- & \\
\hline Provider recommended testing & 5.0 & $1.65-15.2$ & $5.89(1.40-24.9)$ \\
\hline Intend to be tested in 6 months & 3.04 & $1.01-9.17$ & \\
\hline High HIV testing stigma & 0.24 & $0.07-0.82$ & $0.17(0.04-0.77)$ \\
\hline HIV coping self-efficacy $\approx$ & 1.0 & $0.92-1.1$ & \\
\hline \multicolumn{4}{|c|}{$\begin{array}{l}\text { Race variable coded as a categorical variable with White as the reference group }(2=\text { African American, } 1=\text { Hispanic, } 0=\text { non-Hispanic White). SD indicates standard } \\
\text { deviation; UAOR, unadjusted odds ratio of having a positive attitude toward HIV testing; MLR, multiple logistic regression; OR odds ratios; CI, } 95 \% \text { confidence interva } \\
\text { P, p value. } \\
\approx \text { HIV testing stigma was dichotomized at the mean due to the distribution of responses. Whereas HIV coping self-efficacy was used as a continuous scale due to the } \\
\text { normal distribution of responses. } \\
\text { * Factors significant at } 0.10 \text { or less in simple logistic regression and theoretical factors where included in the final model. The final adjusted model was controlled for } \\
\text { pregnancy. Prior HIV testing history was dropped from the final model due to co-linearity. } \\
\text { ¥P value for the final model using multiple logistic regressions. Variables at } \mathrm{p} \text { value }<0.05 \text { included in the final model. } \\
\text { E Range of self-reported number of partners among youth who reported being not sexually active }(0-9) \text {. }\end{array}$} \\
\hline
\end{tabular}

Table 4: Factors Associated with HIV Testing in Never Sexually Active Adolescents $(\mathrm{N}=18,22 \%)$.

\section{Discussion}

A cross-sectional survey of teens seeking care in an urban ambulatory setting, investigating several factors related to HIV testing attitudes and behavior in sexually active youth as well as those with no prior sexual history was conducted. Most teens (regardless of prior sexual history) had a positive attitude toward routine HIV testing. Sexually active youth described a strong ability to cope with a positive diagnosis (high HIV coping self-efficacy) and a positive attitude toward routine testing. However, neither coping self-efficacy nor positive attitude toward HIV testing was associated with receipt of an HIV test at the time of the visit in this population.

This study design does not enable an exploration of why coping self-efficacy and positive attitude was not associated with HIV testing behavior. However, previous work indicates that in urban youth, the role of coping in risky behaviors may be quite complex [14]. Urban teens exhibit varying levels of internalizing and externalizing behavior based on their stress exposure, use of social support, as well as their coping strategies [14]. Therefore, while coping self-efficacy in urban teens is likely to be an influential factor in HIV testing behavior, it may be mediated by other factors such as social support and stress exposure, so that it does not per se yield a strong, independent relationship to testing behavior. Locke demonstrated that HIV testing was associated with stronger general self-efficacy [7] and others have demonstrated that self-efficacy for HIV risk reduction is independently associated with HIV testing among a sample of girls [2]. This suggests that providers should explore coping strategies with patients as it impacts HIV testing attitudes and may ultimately impact behaviors in concert with other counseling strategies.

\section{HIV stigma}

Among youth who were not sexually active, high HIV stigma was associated with a decreased likelihood of being tested at the visit. These findings are consistent with studies demonstrating the role of stigma as a barrier to HIV-related testing and services [5]. The current results place emphasis on the role of stigma for youth who are thought to be at low risk because they have no prior sexual history. This focus is consistent with prior work that found stigma to be more salient to populations who perceive themselves at low-risk [13]. In order for universal, routine HIV testing to be effective, more community-wide interventions, advocacy and education are needed to address the stigma associated with HIV testing. The creation of AIDS-competent communities is one urban-based strategy shown to be effective in dealing with stigma [19].

Provider recommendation significantly impacted HIV testing behavior in sexually active youth. Provider recommendation was also independently associated with HIV testing in youth who reported no prior sexual history. Despite recommendations from the Centers for Disease Control and Prevention, the US Preventive Task Force and the American Academy of Pediatrics for universal HIV testing for teens, [20-22] perceived provider approval and recommendation continues to act as a major factor for teens who choose to accept HIV testing [2325]. This study suggests that teens seeking care in urban settings are more likely to accept HIV testing when they perceive their healthcare providers support the decision for testing and promote testing as part of the visit. Female gender was also associated with receipt of HIV testing, but this finding is similar to other studies suggesting that providers test certain populations of teens who may already be seeking care for other reasons (contraception, STD testing). [26] Thus, providers who continue to use a targeted or risk-based testing approach may neglect at-risk teens who rely on provider recommendation for testing.

The strength of this study is its ability to quantitatively examine how different factors correlate with HIV testing attitudes and behaviors, in an urban community of teens experiencing disproportionately high rates of HIV and risk for HIV acquisition. Future research should establish the generalizability of these findings to other urban domestic and international populations and employ prospective studies to clarify the mechanism between HIV-related stigma and coping strategies used by teens, who choose to accept HIV testing. Strategies should also encourage providers to promote teens to be tested for HIV and prevention strategies may need to improve an adolescent's ability to cope with the perceived psychosocial and physical consequences of HIV infection and address HIV stigma in teens with low perceived risk of HIV in order to be effective. 
Citation: Arrington-Sanders R, Ellen J, Thorpe RJ, Leonard L (2014) What Makes a Teen Get Tested? A Case of Urban Based Sample of Adolescents. J AIDS Clin Res 5: 336. doi:10.4172/2155-6113.1000336

The findings of this work should be examined in light of limitations. Because of the cross-sectional design of this study, analysis was not meant to demonstrate causal relationships between independent and dependent variables. This study's focus on one urban clinic limits the ability to generalize to other adolescent communities at-risk for HIV. Additionally, data was collected six years ago may not reflect current testing behavior. It is unlikely, however, that there have been sufficient structural changes that alter one's ability to cope with a diagnosis since inception of this survey. Data relied upon self-report, but given that youth were surveyed immediately after this visit, any effect of selfreporting is likely small.

Despite these limitations, this study suggests that the ability to cope with a positive diagnosis likely dictates attitudes toward engagement in adolescent HIV testing, but may not be enough to prompt HIV testing behavior. HIV-related stigma is an important factor for urban teens perceived to have little or no risk for HIV acquisition. Provider recommendation may be the most important independent factor for testing teens who live in urban settings, regardless of their sexual history. While it may be discouraging that issues related to coping and stigma inhibit testing, we should take heart that provider recommendation has such a strong effect on the HIV testing behavior of urban teens. Providers should closely examine their HIV testing strategies, for teens both with and without sexual histories, to best serve these urban communities.

\section{Competing Interest}

The authors have no competing interests in the work presented. Each of the authors contributed to the design, analysis and manuscript preparation.

\section{Acknowledgement}

This work was supported by amfAR, the Foundation for AIDS Research Grant (106874-42-RGAT, PI:Leonard) and National Institute of Nursing Research Gran (1R21NR010442PI:Leonard). We would like to thank the research team and Anna Goddu for the work related to this project.

\section{References}

1. Li C, Balluz LS, Okoro CA, Strine TW, Lin JM, et al. (2011) Surveillance of certain health behaviors and conditions among states and selected local areas --- Behavioral Risk Factor Surveillance System, United States, 2009. MMWR Surveill Summ 60: 1-250.

2. Swenson RR, Rizzo CJ, Brown LK, Payne N, DiClemente RJ, et al. (2009) Prevalence and correlates of HIV testing among sexually active African American adolescents in 4 US cities. Sex Transm Dis 36: 584-591.

3. Peralta L, Deeds BG, Hipszer S, Ghalib K (2007) Barriers and facilitators to adolescent HIV testing. AIDS Patient Care STDS 21: 400-408.

4. Scott HM, Pollack L, Rebchook GM, Huebner DM, Peterson J, et al. (2014) Peer social support is associated with recent HIV testing among young black men who have sex with men. AIDS Behav 18: 913-920.

5. Fortenberry JD, McFarlane M, Bleakley A, Bull S, Fishbein M, et al. (2002) Relationships of stigma and shame to gonorrhea and HIV screening. Am J Public Health 92: 378-381.

6. Corrigan PW, Penn DL (1999) Lessons from social psychology on discrediting psychiatric stigma. Am Psychol 54: 765-776.

7. Locke TF, Newcomb MD (2008) Correlates and predictors of HIV risk among inner-city African American female teenagers. Health Psychol 27: 337-348.

8. Bandura A (1994) Self-efficacy. In: Ramachaudran VS, (Ed.), Encyclopedia of Human Behavior. Academic Press, New York 4: 71-81.

9. Eccles JS, Midgley C (1989) State-environment fit: Developmentally appropriate classrooms for young adolescents. In: Ames R, Ames C (Eds.), Research on Motivation in Education: Goals and Cognition. Academic Press, New York, 3: 36-58.
10. Cunningham SD, Kerrigan DL, Jennings JM, Ellen JM (2009) Relationships between perceived STD-related stigma, STD-related shame and STD screening among a household sample of adolescents. Perspect Sex Reprod Health 41: 225-230.

11. Nyamathi AM, Stein JA, Swanson JM (2000) Personal, cognitive, behavioral, and demographic predictors of HIV testing and STDs in homeless women. $J$ Behav Med 23: 123-147.

12. Maedot P, Haile A, Lulseged S, Belachew A (2007) Determinants of vct uptake among pregnant women attending two ANC clinics in Addis Ababa City: unmatched case control study. Ethiop Med J 45: 335-342.

13. Andrinopoulos K, Kerrigan D, Figueroa JP, Reese R, Ellen JM (2010) HIV coping self-efficacy: a key to understanding stigma and HIV test acceptance among incarcerated men in Jamaica. AIDS Care 22: 339-347.

14. Tandon SD, Dariotis JK, Tucker MG, Sonenstein FL (2013) Coping, stress, and social support associations with internalizing and externalizing behavior among urban adolescents and young adults: revelations from a cluster analysis. Adolesc Health 52: 627-633.

15. Gaylord-Harden NK, Gipson P, Mance G, Grant KE (2008) Coping patterns of African American adolescents: A confirmatory factor analysis and cluster analysis of the children's coping strategies checklist. Psychol Assess 20: 10-22.

16. McLoyd VC (1998) Socioeconomic disadvantage and child development. Am Psychol 53: 185-204.

17. DiClemente RJ, Sales JM, Danner F, Crosby RA (2011) Association between sexually transmitted diseases and young adults' self-reported abstinence. Pediatrics 127: 208-213.

18. Maryland Infectious Disease and Environmental Health Administration Surveillence Report.

19. Reed SJ, Miller RL; Adolescent Medicine Trials Network for HIVIAIDS Interventions (2013) Connect to protect and the creation of AIDS-competent communities. AIDS Educ Prev 25: 255-267.

20. Branson BM, Handsfield HH, Lampe MA, Janssen RS, Taylor AW, et al. (2006) Revised recommendations for HIV testing of adults, adolescents, and pregnant women in health-care settings. MMWR Recomm Rep 55: 1-17.

21. Committee on Pediatric AIDS, Emmanuel PJ, Martinez J (2011) Adolescents and HIV infection: the pediatrician's role in promoting routine testing. Pediatrics 128: $1023-1029$

22. Moyer VA, U.S. Preventive Services Task Force (2013) Screening for HIV: U.S Preventive Services Task Force Recommendation Statement. Ann Intern Med 159: 51-60.

23. Kowalczyk Mullins TL, Braverman PK, Dorn LD, Kollar LM, Kahn JA (2010) Adolescent preferences for human immunodeficiency virus testing methods and impact of rapid tests on receipt of results. J Adolesc Health 46: 162-168.

24. Leonard L, Berndtson K, Matson P, Philbin M, Arrington-Sanders R, et al (2010) How physicians test: clinical practice guidelines and HIV screening practices with adolescent patients. AIDS Educ Prev 22: 538-545.

25. Kim EK, Thorpe L, Myers JE, Nash D (2012) Healthcare-related correlates of recent HIV testing in New York City. Prev Med 54: 440-443.

26. Arrington-Sanders R, Ellen J, Trent M (2008) HIV testing in adolescents and young adults receiving STI testing in an urban primary care setting. Sex Transm Dis 35: 686-688. 